\title{
Circular languages generated by complete splicing systems and pure unitary languages *
}

\author{
Paola Bonizzoni \\ Dipartimento di Informatica Sistemistica e Comunicazione \\ Università degli Studi di Milano - Bicocca \\ Viale Sarca 336, 20126 Milano - Italy \\ bonizzoni@disco.unimib.it
}

\author{
Clelia De Felice, Rosalba Zizza \\ Dipartimento di Informatica ed Applicazioni, \\ Università di Salerno, via Ponte Don Melillo, \\ 84084 Fisciano (SA), Italy \\ $\{$ defelice,zizza\}@dia.unisa.it
}

\begin{abstract}
Circular splicing systems are a formal model of a generative mechanism of circular words, inspired by a recombinant behaviour of circular DNA. Some unanswered questions are related to the computational power of such systems, and finding a characterization of the class of circular languages generated by circular splicing systems is still an open problem. In this paper we solve this problem for complete systems, which are special finite circular splicing systems. We show that a circular language $L$ is generated by a complete system if and only if the set $\operatorname{Lin}(L)$ of all words corresponding to $L$ is a pure unitary language generated by a set closed under the conjugacy relation. The class of pure unitary languages was introduced by A. Ehrenfeucht, D. Haussler, G. Rozenberg in 1983, as a subclass of the class of context-free languages, together with a characterization of regular pure unitary languages by means of a decidable property. As a direct consequence, we characterize (regular) circular languages generated by complete systems. We can also decide whether the language generated by a complete system is regular. Finally, we point out that complete systems have the same computational power as finite simple systems, an easy type of circular splicing system defined in the literature from the very beginning, when only one rule is allowed. From our results on complete systems, it follows that finite simple systems generate a class of context-free languages containing non-regular languages, showing the incorrectness of a longstanding result on simple systems.
\end{abstract}

\section{Introduction}

The notion of linear splicing systems was first introduced in [16], where Head modelled a recombinant behaviour of DNA molecules as a particular operation between words in a formal language. Subsequently, circular splicing systems were introduced in [17] along with various open problems related to their computational power. In the circular context, the splicing operation acts on two circular DNA molecules by means of a pair of restriction enzymes as follows. Each of these two enzymes is able to recognize a pattern inside one of these two circular DNA molecules and to cut the molecule in the middle of such a pattern. Two linear molecules are produced and then they are pasted together by the action of ligase enzymes. Thus a new circular DNA sequence is generated [16, 18, 22]. For instance, circular splicing models the integration of a plasmid into the DNA of a host bacteria. A language-theoretic operation can be defined and, depending on whether or not these ligase enzymes substitute the recognized pattern, we have Pixton's definition or Head's and Păun's definition.

\footnotetext{
*Partially supported by the MIUR Project "Mathematical aspects and emerging applications of automata and formal languages" (2007), by the ESF Project “Automata: from Mathematics to Applications (AutoMathA)” (2005-2010), by the 60\% Project "Proprietà strutturali e nuovi modelli di rappresentazione nella teoria dei linguaggi formali" (University of Salerno, 2007 ) and by the $60 \%$ Project "Estensioni della teoria dei linguaggi formali e loro proprietà strutturali” (University of Salerno, 2008).

S. Barry Cooper \& Vincent Danos (eds.): Fifth Workshop on Developments in Computational Models -Computational Models From Nature EPTCS 9, 2009, pp. 22-31 doi 10.4204/EPTCS.9.3

(c) Paola Bonizzoni \& Clelia De Felice \& Rosalba Zizza This work is licensed under the Creative Commons Attribution License.
} 
In the circular context we deal with circular words, i.e., equivalence classes with respect to the conjugacy relation $\sim$, defined by $x y \sim y x$, for $x, y \in A^{*}[20]$. Loosely speaking, a circular word is a word $w$ written on a circle and all the words that are equivalent to $w$ can be obtained by reading the letters on the circle, starting from any point on it. We can then consider sets of circular words or circular languages. In short, a circular splicing system is a triple $(A, I, R)$ where $A$ is a finite alphabet, $I$ is the initial circular language and $R$ is the set of rules, having the form $u_{1} \# u_{2} \$ u_{3} \# u_{4}$, with $u_{i} \in A^{*}, 1 \leq i \leq 4$. Splicing rules are iteratively applied starting from $I$. The corresponding circular splicing language is the smallest language which contains $I$ and is invariant under iterated splicing by rules in $R$. Finding a characterization of the class of circular languages generated by circular splicing systems is still an open problem. Partial results are known for Pixton (resp. Păun) circular splicing systems $S=(A, I, R)$ with $I$ and $R$ satisfying additional assumptions, namely $R$ is assumed to be a reflexive, symmetric set of rules and self-splicing is allowed [18, 23].

In this paper we consider classes of splicing systems where the splicing operation is of a simpler form and for which finding a relationship with regular circular languages is a quite natural question investigated in this research area. Precisely, we consider Păun systems $S$ with both $I$ and $R$ finite sets (finite Păun systems) and such that $a \# 1 \$ b \# 1 \in R$, for each $a, b \in A$, where 1 is the empty word and $A$ is the alphabet of $I$. In this case the splicing operation is very easy: it applies to any pair of circular words, $\sim x a, \sim y b$, and gives as a result the circular word $\sim x a y b$. Therefore, the splicing operation can be seen as a concatenation followed by a closure under the conjugacy relation. We have called these systems complete.

The main contribution of this paper is the characterization of the computational power of complete systems. Indeed, we show that a circular language $L$ is generated by a complete system if and only if $\operatorname{Lin}(L)$, the set of all words such that the corresponding circular words are in $L$, is a pure unitary language generated by a set closed under the conjugacy relation.

The class of pure unitary languages is a subclass of the class of context-free languages, introduced in [12]. Equivalently, a language is pure unitary if it is obtained by applying the iterated insertion operation, starting with a finite set $Y$ [15]. Thus we show that circular splicing and iterated insertion are closely related. In the same paper [12], the authors also characterized regular pure unitary languages by means of a decidable property. As a consequence, we characterize regular circular languages generated by complete systems and we can also decide whether a complete system generates a regular circular language.

We also show that complete systems have the same computational power as circular simple systems with only one rule, i.e., finite Păun systems with $R=\{a \# 1 \$ a \# 1\}, a \in A$. As a consequence, we characterize (regular) circular languages generated by circular simple systems with only one rule. The special class of simple systems was first considered in [24] using Head's definition and then in [8] by taking into account Păun's definition, as the circular counterpart of the case of the linear splicing investigated in [21]. In [24], the authors claimed that Head simple systems always generate regular circular languages. In this paper we show that there are simple systems generating non-regular context-free circular languages and we give new contributions towards the solution of the still open problem of finding a characterization of the class of circular languages generated by finite circular simple splicing systems. (As a matter of fact, an example of a non-regular circular language generated by a simple system has been also reported in [5], see Example 4.4).

A still unanswered question is whether the above-mentioned characterization of regularity can be generalized to larger classes of circular splicing languages, in particular to the class of simple systems.

The paper is organized as follows. In Section 2 we gathered basics on (circular) words and languages, circular splicing, CSSH-systems and simple systems. We also conclude the section with a short descrip- 
tion of how state of the art the open problems are. In Section 3 we state some known and new results on pure unitary languages. Section 4 is devoted to our main results. All the proofs have been omitted for space constraint but they can be found in an extended version of this paper [6]. The main results of this paper were also communicated at AutoMathA 2009 (Liege, Belgium, 8-12 June 2009).

\section{Basics}

\section{1 (Circular) words and languages}

We denote by $A^{*}$ the free monoid over a finite alphabet $A$ and we set $A^{+}=A^{*} \backslash 1$, where 1 is the empty word. For a word $w \in A^{*},|w|$ is the length of $w$ and $|w|_{a}$ is the number of occurrences of $a$ in $w, a \in A$. We also set alph $(w)=\left\{\left.a \in A|| w\right|_{a}>0\right\}$. A word $x \in A^{*}$ is a factor of $w \in A^{*}$ if $u_{1}, u_{2} \in A^{*}$ exist such that $w=u_{1} x u_{2}$. For a subset $Y$ of $A^{*},|Y|$ is the cardinality of $Y$ and $\operatorname{alph}(Y)=\cup_{y \in Y} \operatorname{alph}(y)$. We denote by Fin (resp. Reg) the class of finite (resp. regular) languages over $A$. Given $w \in A^{*}$, a circular word $\sim w$ is the equivalence class of $w$ with respect to the conjugacy relation $\sim$ defined by $x y \sim y x$, for $x, y \in A^{*}[20]$. The notations $|\sim w|,|\sim w|_{a}, \operatorname{alph}(\sim w)$ will be defined as $|w|,|w|_{a}, \operatorname{alph}(w)$, for any representative $w$ of $\sim w$. We will often use the notation $w$ for a circular word $\sim w$. Let $\sim A^{*}$ denote the set of all circular words over $A$, i.e., the quotient of $A^{*}$ with respect to $\sim$. Given $L \subseteq A^{*}, \sim L=\{\sim w \mid w \in L\}$ is the circularization of $L$ whereas, given a circular language $C \subseteq \sim A^{*}$, every $L \subseteq A^{*}$ such that $\sim L=C$ is a linearization of $C$. In particular, a linearization of $\sim w$ is a linearization of $\{\sim w\}$, whereas the full linearization $\operatorname{Lin}(C)$ of $C$ is defined by $\operatorname{Lin}(C)=\left\{w \in A^{*} \mid \sim_{w} \in C\right\}$. We will often write ${ }^{\sim} w$ instead of $\{\sim w\}$ and $L$ instead of $\sim L$, for a set of letters $L \subseteq A$. Given a family of languages $F A$ in the Chomsky hierarchy, $F A^{\sim}$ is the set of all those circular languages $C$ which have some linearization in $F A$. Thus, $\operatorname{Reg}^{\sim}=\left\{C \subseteq \sim A^{*} \mid \exists L \in \operatorname{Reg}: \sim L=C\right\}$. If $C \in \operatorname{Reg}^{\sim}$ then $C$ is a regular circular language. It is classically known that given a regular (resp. context-free) language $L \subseteq A^{*}, \operatorname{Lin}(\sim L)$ is regular (resp. context-free) [19]. As a result, given $C \subseteq \sim A^{*}$, we have $C \in \operatorname{Reg}^{\sim}$ (resp. $C$ is a context-free circular language) if and only if $\operatorname{Lin}(C)$ is regular (resp. context-free) [18].

\subsection{Circular splicing}

In this paper we deal with the definition of the circular splicing operation given in [18]. The corresponding circular splicing systems are named here Păun circular splicing systems since they are the counterpart of Păun linear splicing systems in the circular context.

Păun's definition [18]. A Păun circular splicing system is a triple $S=(A, I, R)$, where $A$ is a finite alphabet, $I$ is the initial circular language, with $I \subseteq \sim A^{*}$ and $R$ is the set of the rules, with $R \subseteq A^{*} \# A^{*} \$ A^{*} \# A^{*}$ and $\#, \$ \notin A$. Then, given a rule $r=u_{1} \# u_{2} \$ u_{3} \# u_{4}$ and circular words $\sim_{w^{\prime}}, \sim_{w^{\prime \prime}}, \sim_{w}$, we set $\left(\sim^{\prime}, \sim w^{\prime \prime}\right) \vdash_{r} \sim w$ if there are linearizations $w^{\prime}$ of $\sim w^{\prime}, w^{\prime \prime}$ of $\sim w^{\prime \prime}, w$ of $\sim_{w}$ such that $w^{\prime}=u_{2} x u_{1}$, $w^{\prime \prime}=u_{4} y u_{3}$ and $w=u_{2} x u_{1} u_{4} y u_{3}$. If $\left(\sim^{\prime}, \sim w^{\prime \prime}\right) \vdash_{r} \sim w$ we say that $\sim_{w}$ is generated (or spliced) starting with $\sim w^{\prime}, \sim w^{\prime \prime}$ and by using a rule $r$. We also say that $u_{1} u_{2}, u_{3} u_{4}$ are sites of splicing.

We recall that in the original definition of circular splicing given in [18], rules in $R$ could be used in two different ways. One way has been described above, while the other, known as self-splicing, will not be considered here. From now on, "splicing system" will be synonymous with "circular splicing system".

Given a splicing system $S$ and a circular language $C \subseteq \sim A^{*}$, we set $\sigma^{\prime}(C)=\left\{w \in \sim A^{*} \mid \exists w^{\prime}, w^{\prime \prime} \in\right.$ $\left.C, \exists r \in R:\left(w^{\prime}, w^{\prime \prime}\right) \vdash_{r} w\right\}$. We also define $\sigma^{0}(C)=C, \sigma^{i+1}(C)=\sigma^{i}(C) \cup \sigma^{\prime}\left(\sigma^{i}(C)\right), i \geq 0$, and $\sigma^{*}(C)=$ 
$\bigcup_{i \geq 0} \sigma^{i}(C)$. Then, $L(S)=\sigma^{*}(I)$ is the circular language generated by $S$. A circular language $C$ is $P \breve{u} u n$ generated (or $C$ is a circular splicing language) if a splicing system $S$ exists such that $C=L(S)$. In the sequel $C($ Fin, Fin $)$ denotes the class of the circular languages generated by finite Păun splicing systems, where $S=(A, I, R)$ is a finite splicing system if $I$ and $R$ are both finite sets.

As observed in [18], we may assume that the set $R$ of the rules in a splicing system $S=(A, I, R)$ satisfies additional conditions, having also a biological counterpart. Namely, we may assume that $R$ is reflexive (i.e., for each $u_{1} \# u_{2} \$ u_{3} \# u_{4} \in R$, we have $u_{1} \# u_{2} \$ u_{1} \# u_{2}, u_{3} \# u_{4} \$ u_{3} \# u_{4} \in R$ ) or $R$ is symmetric (i.e., for each $u_{1} \# u_{2} \$ u_{3} \# u_{4} \in R$, we have $u_{3} \# u_{4} \$ u_{1} \# u_{2} \in R$ ). We do not assume that $R$ is reflexive. On the contrary, we notice that, in view of the definition of circular splicing, if $\left(w^{\prime}, w^{\prime \prime}\right) \vdash_{r} w$, with $r=u_{1} \# u_{2} \$ u_{3} \# u_{4}$, then $\left(w^{\prime \prime}, w^{\prime}\right) \vdash_{r^{\prime}} w$, with $r^{\prime}=u_{3} \# u_{4} \$ u_{1} \# u_{2}$. Consequently, $L(S)=L\left(S^{\prime}\right)$, where $S^{\prime}=$ $\left(A, I, R^{\prime}\right)$ and $R^{\prime}=R \cup\left\{u_{3} \# u_{4} \$ u_{1} \# u_{2} \mid u_{1} \# u_{2} \$ u_{3} \# u_{4} \in R\right\}$. Hence, in order to find a characterization of the circular splicing languages, there is no loss of generality in assuming that $R$ is symmetric. Thus, in what follows, we assume that $R$ is symmetric. However, for simplicity, in the examples of Păun systems, only one of either $u_{1} \# u_{2} \$ u_{3} \# u_{4}$ or $u_{3} \# u_{4} \$ u_{1} \# u_{2}$ will be reported in the set of rules.

As already said, in this paper we consider some special classes of finite circular splicing systems. In detail, a Păun circular semi-simple splicing system (CSSH system for short) is a finite splicing system $S=(A, I, R)$ such that, for each $u_{1} \# u_{2} \$ u_{3} \# u_{4} \in R$, we have $\left|u_{1} u_{2}\right|=\left|u_{3} u_{4}\right|=1$ [7]. CSSH systems have been considered in [7, 8, 24], once again as the circular counterpart of linear semi-simple splicing systems introduced in [14]. If $u_{1} u_{2}=u_{3} u_{4} \in A$ then $S$ is a simple system [8]. Thus, there are four types of rules, namely $a_{i} \# 1 \$ a_{j} \# 1, a_{i} \# 1 \$ 1 \# a_{j}, 1 \# a_{i} \$ a_{j} \# 1$ and $1 \# a_{i} \$ 1 \# a_{j}$, with $a_{i}, a_{j} \in A$. Furthermore, since $R$ is symmetric, if $a_{i} \# 1 \$ 1 \# a_{j} \in R$ then we also have $1 \# a_{j} \$ a_{i} \# 1 \in R$. So, using the terminology of [7, 8], a (1,3)-CSSH system (resp. (1,3)-circular simple system) is a CSSH system (resp. circular simple system) where each rule has the form $a_{i} \# 1 \$ a_{j} \# 1$, with $a_{i}, a_{j} \in A$. A (2,3)-CSSH system (resp. (2,3)circular simple system) is a CSSH system (resp. circular simple system) where each rule has the form $1 \# a_{i} \$ a_{j} \# 1$, with $a_{i}, a_{j} \in A$. Finally, a $(2,4)$-CSSH system (resp. $(2,4)$-circular simple system) is a CSSH system (resp. circular simple system) where each rule has the form $1 \# a_{i} \$ 1 \# a_{j}$, with $a_{i}, a_{j} \in A$. Notice that in a $(1,3)-\mathrm{CSSH}$ system, circular splicing can be rephrased as follows: given a rule $a_{i} \# 1 \$ a_{j} \# 1$ and two circular words $\sim x a_{i}, \sim y a_{j}$, the circular splicing yields as a result $\sim x a_{i} y a_{j}$. In what follows and in order to abbreviate, we will write $\left(a_{i}, a_{j}\right)$ to denote a rule $a_{i} \# 1 \$ a_{j} \# 1$ in a $(1,3)$-CSSH system. We will focus on a special subclass of these systems, defined in Section 4 and named complete systems.

\subsection{State of the art}

In this section we will give a brief description of how state of the art the open problems on circular splicing are. The main result concerning the computational power of circular splicing systems states that if $S=(A, I, R)$ is a Păun circular splicing system such that $I \in F A^{\sim}$, where $F A$ is a full abstract family of languages which is closed under cyclic closure (i.e., if $L \in F A$ then $\operatorname{Lin}(\sim L) \in F A$ ), $R$ is a finite, reflexive and symmetric set of rules and self-splicing is used, then $L(S) \in F A^{\sim}$ [18]. Other results have been proved for finite circular splicing systems [2, 3, 4]. In particular, it is known that $C$ (Fin,Fin) contains regular circular languages (see [3]), context-free circular languages which are not regular (see [3, 24]), context-sensitive circular languages which are not context-free (see [13]) and there exist regular circular languages which are not in $C($ Fin, Fin) (see [3] $)$. However, the problem of characterizing circular languages in $\operatorname{Reg}^{\sim} \cap C($ Fin,Fin) remains open. We do not even know if, given $L$ in $C$ (Fin,Fin), it is decidable whether $L$ is regular. A characterization of $C($ Fin, Fin) (and of $\operatorname{Reg} \sim \cap C($ Fin,Fin) ) has been obtained for languages over a one-letter alphabet in [3, 4].

Concerning CSSH systems, in [8], the authors compared the classes of circular languages generated 
by $(i, j)$-circular simple systems, for different values of $(i, j) \in\{(1,3),(2,4),(2,3)\}$, i.e., for different positions of the letter in the rule. They proved that the class of circular languages generated by $(1,3)$-circular simple systems (resp. (2,3)-circular simple systems) is equal to the class of languages generated by $(2,4)$-circular simple systems (resp. $(1,4)$-circular simple systems), whereas the class of circular languages generated by $(1,3)$ and $(2,3)$-circular simple systems are not comparable. An analogous viewpoint was adopted for Păun circular semi-simple splicing systems in [7] where the authors highlighted further differences between circular simple and CSSH systems. Indeed, they proved that the class of circular languages generated by $(1,3)-\mathrm{CSSH}$ systems and the class of circular languages generated by $(2,4)-\mathrm{CSSH}$ systems are not comparable. Finally, in [10] the authors proved that the class of circular languages generated by $(2,4)-\mathrm{CSSH}$ systems is the class of the reversal of the circular languages generated by $(1,3)$-CSSH systems. Loosely speaking, the reversal of a (circular) word is the (circular) word written backwards and the reversal of a (circular) language $L$ is the (circular) language consisting of the reversals of all its (circular) words.

In [13], the author claimed that the class of circular languages generated by CSSH systems is contained in the class of context-free circular languages. Marked systems, i.e., CSSH systems satisfying additional hypotheses, were introduced in [9, 10] with a characterization of the corresponding regular circular languages generated. This characterization was reviewed in a graph theoretical setting in [5]. However, a still open problem is to find a characterization of the class of regular circular languages generated by CSSH systems.

Remark 2.1 As stated in the introduction, the notion of simple splicing systems has been originally considered using the Head splicing operation. A Head circular splicing system $S_{H}=(A, I, T, P)$ is defined by giving a finite alphabet $A$, the initial set $I \subseteq \sim A^{*}$, the set $T$ of triples, $T \subseteq A^{*} \times A^{*} \times A^{*}$, and where $P$ is a binary relation on $T$ such that, for each $(p, x, q),(u, y, v) \in T,(p, x, q) P(u, y, v)$ if and only if $x=y$. Head defined circular splicing as an operation on two circular words $\sim h p x q, \sim$ kuxv $\in \sim A^{*}$ performed by two triples $(p, x, q),(u, x, v)$ and producing $\sim h p x v k u x q$. The word $x$ is called a crossing of the triple. In [3], the authors proved that there is a canonical transformation of a Head system $S_{H}=(A, I, T, P)$ into a Păun system $S=(A, I, R)$ such that $S$ and $S_{H}$ generate the same language. This transformation is defined by $R=\{p x \# q \$ u x \# v \mid(p, x, q),(u, x, v) \in T\}$ (Proposition 3.1 in [3]). We notice that this transformation defines a bijection between the class of the circular languages generated by $(1,3)$-circular simple systems $S=(A, I, R)$, with $R=\{(a, a)\}$ and that of circular languages generated by Head splicing systems $S_{H}=(A, I, T, P)$ with $T=\{(1, a, 1)\},(1, a, 1) P(1, a, 1)$. On the other hand, in [24], the authors discussed Head systems $S_{H}=(A, I, T, P)$ and the corresponding splicing operation was named action $S A 1$ of $S_{H}$. Furthermore, a triple in $T$ of the form $(1, a, 1), a \in A$, was said to have null context and crossing of length one. The first part of Theorem 3.4 in [24] claims the regularity of the circular splicing language under action SA1 of a splicing system $S_{H}=(A, I, T, P)$ with $I$ finite and triples in $T$ having null context and crossings of length one. On the contrary, Example 4.4 shows that $(1,3)$-circular simple systems $S$ exist with $L(S)$ being a non-regular language, so disproving the above-mentioned Theorem 3.4 in [24].

\section{Pure unitary languages}

In this section, we deal with a class of languages, named pure unitary languages, already considered in [11, 12, 15] with the aim of finding conditions under which a context-free grammar will generate a regular language. Pure unitary languages (named insertion languages in [11]) are a simple class of "generalized Dyck languages" and they can be defined in several ways. Here we follow the viewpoint 
adopted in [15], where these languages are defined by means of the operation of iterated insertion. We report some of their known properties and we also give a condition under which a pure unitary language is closed under the conjugacy relation (Lemma 3.3). The operations of insertion and iterated insertion are variants of classical operations on formal languages. We recall their definitions below.

Definition 3.1 [15] Given $Z, Y \subseteq A^{*}$, the operation of insertion, denoted by $\leftarrow$, is defined by $Z \leftarrow Y=$ $\left\{z_{1} y z_{2} \mid z_{1} z_{2} \in Z\right.$ and $\left.y \in Y\right\}$. The operation of iterated insertion, denoted by $\leftarrow_{*}$, is defined inductively from the operation of insertion by $Y^{\leftarrow 0}=\{1\}, Y^{\leftarrow i+1}=Y^{\leftarrow i} \leftarrow Y$ and $Y^{\leftarrow *}=\cup_{i \geq 0} Y^{\leftarrow i}$.

Definition 3.2 [12] 15] A language $L$ is a pure unitary language if $L=Y^{\leftarrow *}$ with $Y$ being a finite set.

Since $Y^{\leftarrow_{*}}=(Y \backslash 1)^{\leftarrow_{*}}$, in what follows, we assume $Y \subseteq A^{+}$. In [12] the authors stated a characterization of regular pure unitary languages by means of a decidable property. This result is partially reported below.

Theorem 3.1 [12] Let $Y$ be a finite set such that alph $(Y)=A$. Then $L=Y^{\leftarrow *}$ is regular if and only if $Y$ is subword unavoidable in $A^{*}$, i.e., there exists a positive integer $k$ such that any word $u \in A^{*}$, with $|u| \geq k$, contains as a factor a word of $Y$. For any regular set $R \subseteq A^{*}$, it is decidable whether or not $R$ is subword unavoidable in $A^{*}$.

The construction of a grammar generating $Y^{\leftarrow *}$ is folklore and is reported below. As usual, here and from now on we denote by $L(G)$ the language generated by a context-free grammar $G$. Let $w=$ $a_{i_{1}} \cdots a_{i_{h}} \in Y$, where $\left\{a_{i_{1}}, \ldots, a_{i_{h}}\right\}$ is a multiset of elements in $A$. Then, we define the production $p_{w}=$ $X \rightarrow X a_{i_{1}} X a_{i_{2}} \cdots X a_{i_{h}} X$. We set $G_{Y}=(\{X\}, A, P, X), P=\{X \rightarrow 1\} \cup\left\{p_{w} \mid w \in Y\right\}$ and we shall call $G_{Y}$ the pure unitary grammar associated with $Y$. Our main result follows from the relation $Y^{\leftarrow *}=$ $L\left(G_{Y}\right)$ (Proposition 3.1 folklore) and from two properties of the grammar $G_{Y}$, namely the closure of the language $L\left(G_{Y}\right)$ under concatenation (Lemma 3.2) and under the conjugacy relation (Lemma 3.3), the latter property being satisfied under the condition that $Y$ keeps the same property. Lemma 3.1 is needed in the proof of Lemma 3.3 and the three lemmas show a not yet thoroughly investigated relation between insertion, concatenation and closure under the conjugacy relation.

Lemma 3.1 For each $w, z \in L\left(G_{Y}\right)$, for each $w_{1}, w_{2} \in A^{*}$ such that $w=w_{1} w_{2}$, we have $w_{1} z w_{2} \in L\left(G_{Y}\right)$.

Proposition 3.1 Given a finite set $Y \subseteq A^{*}$, we have $Y^{\leftarrow *}=L\left(G_{Y}\right)$.

The following two lemmas are needed in the next section.

Lemma 3.2 Given a finite set $Y \subseteq A^{*}$, the language $Y^{\leftarrow *}$ is closed under concatenation, i.e., if $w_{1}, w_{2} \in$ $Y^{\leftarrow *}$ then $w_{1} w_{2} \in Y^{\leftarrow *}$.

Lemma 3.3 Let $Y$ be a finite set. If $Y$ is closed under the conjugacy relation then $L\left(G_{Y}\right)$ is closed under the conjugacy relation.

\section{Main Result}

In this section we will state the main results of the paper. Precisely, in Section 4.1 we introduce the notion of a complete system $S$ and we state that $L$ is generated by $S$ if and only if $\operatorname{Lin}(L)$ may be obtained by iterated insertion starting with a language closed under the conjugacy relation (Theorem 4.1). A regularity characterization of splicing languages generated by complete systems follows by the above result (Corollary 4.2). Then, in Section 4.2 we discuss the case of (1,3)-circular simple systems with only one rule $(a, a)$. We show that these systems have the same computational power as complete systems in Proposition 4.2. Consequently we characterize the corresponding generated languages. 


\subsection{Complete circular splicing systems}

Definition 4.1 $A$ complete system $S=(A, I, R)$ is a (1,3)-CSSH system such that $R=A \times A$, alph $(I)=A$ and $1 \notin I$.

Example 4.1 Let $S=(A, I, R)$, where $A=\{a, b\}, I=\sim\{a b\}$ and $R=\{(a, a),(b, b),(a, b)\}$. Therefore, $S$ is a complete system. By using Theorem 4.1, we will show that $L(S)$ is a non-regular circular language. On the contrary, let $S=\left(A, I^{\prime}, R\right)$, where $I^{\prime}=\sim\{a b, a a, b b\}$. As we will see below, $S$ is a complete system generating a regular circular language.

Remark 4.1 Let $S=(A, I, R)$ be a complete system. Notice that $1 \notin L(S)$ since, as shown in [3], for any Păun circular splicing system $S=(A, I, R)$, we have that $1 \in I$ if and only if $1 \in L(S)$.

One of our main results, stated in Theorem 4.1, is based on the following observation. On the one hand we have already pointed out that if $L=Y^{\leftarrow *}$ is obtained by iterated insertion, starting with a finite language $Y$ closed under the conjugacy relation, then $L$ is closed under concatenation and under the conjugacy relation (Lemmas 3.2, 3.3). On the other hand, by definition the full linearization $\operatorname{Lin}(L(S)$ ) of a circular splicing language $L(S)$ is a language closed under the conjugacy relation. In addition, if $S$ is a complete system then $\operatorname{Lin}(L(S))$ is also closed under concatenation (Lemma4.1).

Lemma 4.1 Let $S$ be a complete system. Then $\operatorname{Lin}(L(S))$ is closed under concatenation, i.e., if $w, w^{\prime} \in$ $\operatorname{Lin}(L(S))$ then $w w^{\prime} \in \operatorname{Lin}(L(S))$. Analogously, if $\sim w, \sim_{w^{\prime}} \in L(S)$ then $\sim w w^{\prime} \in L(S)$.

The above result is strengthened by Proposition 4.1. Indeed we state that $\operatorname{Lin}(L(S))$ is the smallest language closed under conjugacy relation, under concatenation and containing $I$.

Proposition 4.1 Let $S=(A, I, R)$ be a complete system. Let $L$ be a language such that $L$ is closed under the concatenation, $L$ is closed under the conjugacy relation and $\operatorname{Lin}(I) \subseteq L$. Then $\operatorname{Lin}(L(S)) \subseteq L$.

Since the language $L=(\operatorname{Lin}(I))^{\leftarrow *}$, obtained by iterated insertion starting with $\operatorname{Lin}(I)$, satisfies the hypotheses in Proposition 4.1, we obtain Corollary 4.1 as a direct result.

Corollary 4.1 Let $S=(A, I, R)$ be a complete system. Then $\operatorname{Lin}(L(S)) \subseteq(\operatorname{Lin}(I))^{\leftarrow *} \backslash 1$.

By the previous preliminary results we can thus prove Theorem 4.1 stating the connection between pure unitary languages, pure unitary grammars and circular splicing languages.

Theorem 4.1 The following conditions are equivalent:

(1) There exists a complete system $S=(A, I, R)$ such that $L=L(S)$.

(2) There exists a finite language $Y$, with alph $(Y)=A$, such that $Y$ is closed under the conjugacy relation and $\operatorname{Lin}(L)=L\left(G_{Y}\right) \backslash 1$, i.e., $L=\sim\left(L\left(G_{Y}\right) \backslash 1\right)$.

(3) There exists a finite language $Y$, with alph $(Y)=A$, such that $Y$ is closed under the conjugacy relation and $\operatorname{Lin}(L)=Y^{\leftarrow *} \backslash 1$, i.e., $L=\sim\left(Y^{\leftarrow *} \backslash 1\right)$.

Example 4.2 Let $S=(A, I, R)$ be the complete system reported in Example 4.1, i.e., $A=\{a, b\}, I=$ $\sim\{a b\}$ and $R=\{(a, a),(b, b),(a, b)\}$. By using Theorem 4.1 we have $\operatorname{Lin}(L(S))=\left\{\left.w \in\{a, b\}^{+}|| w\right|_{a}=\right.$ $\left.|w|_{b}\right\}$. Thus $\operatorname{Lin}(L(S))$ is a non-regular language and consequently $L(S)$ is a non-regular circular language.

A fundamental consequence of Theorems 3.1 and 4.1 is stated in Corollary 4.2 
Corollary 4.2 Let $S=(A, I, R)$ be a complete system. Then, $L(S)$ is a regular circular language if and only if $\operatorname{Lin}(I)$ is subword unavoidable. Consequently, it is decidable whether $L(S)$ is a regular circular language.

Example 4.3 Let $S=\left(A, I^{\prime}, R\right)$ be the complete system reported in Example 4.1, i.e., $A=\{a, b\}, I^{\prime}=$ $\sim\{a b, a a, b b\}$ and $R=\{(a, a),(b, b),(a, b)\}$. Of course $\operatorname{Lin}\left(I^{\prime}\right)$ is subword unavoidable. Thus, in view of Corollary 4.2, $L(S)$ is a regular circular language. Actually, $\operatorname{Lin}(L(S))=\left(\{a, b\}^{2}\right)^{+}=\left\{w \in\{a, b\}^{*} \mid \exists k>\right.$ $0:|w|=2 k\}$.

Remark 4.2 Let $S$ be a complete system. Thus $L=\operatorname{Lin}(L(S))=\operatorname{Lin}(L(S))^{+}(\operatorname{Lemma} 4.1)$ and $L \cup\{1\}$ is a star language, i.e., $L \cup\{1\}$ is a language which is closed under the conjugacy relation and which is the Kleene closure of a language [3]. In [3], the authors defined a class of regular star languages $L$ by means of a property of the finite state automaton recognizing $L$ and such that $\sim L \in C($ Fin, Fin). Finding the relation between this class of star languages and pure unitary languages is a still unexplored direction.

\subsection{Simple systems}

In this section we show how our characterization of the computational power of complete systems, can be extended to a special type of $(1,3)$-circular simple systems. In detail, a reformulation of Theorem 4.1 and Corollary 4.2 still hold in terms of $(1,3)$-circular simple systems having only one rule. This reformulation is obtained by using a bijection between these systems and complete systems (Proposition 4.2). It is clear that, in order to extend this bijection to $(1,3)$-circular simple systems with a set of rules of larger size, we need a generalization of the notion of complete systems.

Let us briefly give an intuitive description of the results stated in this section. As already said, the circular splicing language is obtained by iterated applications of the splicing operation, starting with all pairs of circular words in $I$. Let $S=(A, I, R)$ be a $(1,3)$-circular simple system where $R=\{(a, a)\}, a \in A$. In this context, given two circular words $\sim h a, \sim k a$, the circular splicing yields as a result $\sim h a k a$. In other words the splicing operation is allowed on every position where $a$ appears. Therefore we handle all words $w$ in $\operatorname{Lin}(I)$ (and in $\operatorname{Lin}\left(\sigma^{i}(I)\right)$ ) having the form $w=x_{1} a \cdots x_{k} a$, where $x_{j} \in(A \backslash a)^{*}$. It is easily seen that each of these words $w$ is in the free monoid generated by a finite prefix code $F=\left\{x_{1} a, \ldots, x_{k} a, \ldots\right\}$. Therefore, by a coding morphism $\alpha_{j} \rightarrow x_{j} a, F$ is identified with a finite alphabet $A^{\prime}$ and the rule $(a, a)$ is identified with the set of rules $A^{\prime} \times A^{\prime}$. Since regular (resp. context-free) languages are closed under morphism, we can define a bijection between complete systems $S^{\prime}$ and (1,3)-circular simple systems $S$ with one rule such that $L\left(S^{\prime}\right)$ is regular (resp. context-free) if and only if $L(S)$ is regular (resp. contextfree). Let us state the definitions and results in a precise way.

Given $I=\sim\left\{w_{1}, \ldots, w_{k} \mid 1 \leq i \leq k\right\}$, the set $F \subseteq A^{+}$associated with $I$ is the set of smallest cardinality which satisfies the following conditions: (1) for each word $w$ in $F$ we have $w \in A^{*} a$ and $|w|_{a}=1$; (2) for each $\sim_{w} \in I$, if $w^{\prime}$ is any linearization of $\sim w$ such that $w^{\prime} \in A^{*} a$ then $w^{\prime} \in F^{+}$.

Hence $F=\left\{x_{i} a \mid 1 \leq i \leq n\right\}$ is a finite prefix code (i.e., $F \cap F A^{+}=\emptyset$ ) and for each ${ }^{\sim} w_{i} \in I$, we have $\sim_{w_{i}}=\sim_{i, 1} a \cdots x_{i, j_{i}} a$, where $j_{i} \in \mathbb{N},\left|x_{i, g}\right|_{a}=0$, for each $1 \leq g \leq j_{i}$ and $x_{i, 1} a, \ldots, x_{i, j_{i}} a$ is a set of not necessarily different elements in $F$. Here, we assume that $a \in \operatorname{alph}(w)$, for each $w \in I$. Notice that, in order to characterize the circular splicing language generated by $S$, there is no loss of generality in making this assumption. Indeed, by using a result stated in [10], we have that $L(S)=L\left(S_{1}\right) \cup\left(I \backslash I_{1}\right)$, where $S_{1}=\left(A, I_{1}, R\right)$ and $I_{1}=\{w \in I \mid a \in a l p h(w)\}$. From now on, $S=(A, I, R)$ will denote a $(1,3)$ circular simple system with $R=\{(a, a)\}, a \in A$ and $I=\sim\left\{x_{i, 1} a \cdots x_{i, j_{i}} a \mid 1 \leq i \leq k\right\}$, where $\left|x_{i, g}\right|_{a}=0$, for each $1 \leq g \leq j_{i}$. Of course $L(S) \subseteq \sim F^{+}$. We now state that the above systems have the same computational power as special complete systems. 
Let $\varphi$ be a coding morphism for $F$, i.e., a morphism $\varphi: A^{* *} \rightarrow A^{*}$ which is injective and such that $F=\varphi\left(A^{\prime}\right)[1]$. Thus, $\left|A^{\prime}\right|=|F|$. Set $A^{\prime}=\left\{\alpha_{1}, \ldots, \alpha_{n}\right\}$. We know that $\varphi$ is defined by $\varphi\left(\alpha_{i}\right)=x_{i} a$, $1 \leq i \leq n$. We also know that $\varphi$ defines a map from $\sim A^{\prime *}$ into $\sim A^{*}$ if we set $\varphi(\sim w)=\sim \varphi(w)$, for all $w \in A^{\prime *}$, i.e., $\varphi\left(\sim_{w}\right)$ does not depend on which representative in $\sim w$ we choose to define it by [18]. Thus, if $w, w^{\prime} \in A^{\prime *}$ and $w \sim w^{\prime}$ then $\varphi(w) \sim \varphi\left(w^{\prime}\right)$. In what follows $S^{\prime}=\left(A^{\prime}, I^{\prime}, R^{\prime}\right)$ will denote the complete system defined by $I^{\prime}=\varphi^{-1}(I)$. Thus, $A^{\prime}=\operatorname{alph}\left(I^{\prime}\right)$ and $R^{\prime}=A^{\prime} \times A^{\prime}$.

Proposition 4.2 We have $\varphi\left(L\left(S^{\prime}\right)\right)=L(S)$ and $\varphi^{-1}(L(S))=L\left(S^{\prime}\right)$. Consequently, $L(S)$ is a regular (resp. context-free) circular language if and only if $L\left(S^{\prime}\right)$ is regular (resp. context-free).

In view of Proposition 4.2, Theorem 4.1 and Corollary 4.2 may be rephrased for $(1,3)$-circular simple systems $S$ with only one rule (see [6]).

Example 4.4 We recall that a word $x \in A^{+}$is called unbordered if $x \in u A^{+} \cap A^{+} u$ implies $u=1$ [1]. Given $w, x \in A^{*}$, with $x$ being an unbordered word, we denote by $|w|_{x}$ the number of occurrences of $x$ in $w$. Let $S=(A, I, R)$ be the $(1,3)$-circular simple system defined by $A=\{a, b, c\}, I=\sim\{$ baca $\}$ and $R=\{(a, a)\}$. In view of Proposition 4.2 we have $L(S)=\varphi\left(L\left(S^{\prime}\right)\right)$, where $S^{\prime}=\left(A^{\prime}, I^{\prime}, R^{\prime}\right)$ is the complete system defined by $I^{\prime}=\sim\{\alpha \beta\}, A^{\prime}=\operatorname{alph}\left(I^{\prime}\right)=\{\alpha, \beta\}$ and $R^{\prime}=A^{\prime} \times A^{\prime}$. By using Theorem 4.1, we have $\operatorname{Lin}\left(L\left(S^{\prime}\right)\right)=\left\{\left.w \in\{\alpha, \beta\}^{+}|| w\right|_{\alpha}=|w|_{\beta}\right\}$ and so $L\left(S^{\prime}\right)=\sim\left\{\left.w \in\{\alpha, \beta\}^{+}|| w\right|_{\alpha}=|w|_{\beta}\right\}$ is a (non-regular) context-free circular language (see Example 4.2). By using Proposition 4.2 once again, $L(S)=\varphi\left(L\left(S^{\prime}\right)\right)=\sim\left\{\left.w \in\{a, b, c\}^{+}|| w\right|_{b a}=|w|_{c a}\right\}$ is a (non-regular) context-free circular language. An ad hoc proof of the non-regularity of the circular language $L(S)$ has also been reported in [5].

\section{ACKNOWLEDGEMENTS}

The authors are very grateful to an anonymous referee for pointing out reference [12].

\section{References}

[1] J. Berstel, D. Perrin, C. Reutenauer, Codes and Automata, Cambridge University Press (2009).

[2] P. Bonizzoni, C. De Felice, G. Mauri, R. Zizza, DNA and circular splicing, in: (A. Condon, G. Rozenberg, Eds.) Proc. DNA 2000, Lecture Notes in Computer Science 2054 (2001) 117-129.

[3] P. Bonizzoni, C. De Felice, G. Mauri, R. Zizza, Circular splicing and regularity, Theoretical Informatics and Applications 38 (2004) 189-228.

[4] P. Bonizzoni, C. De Felice, G. Mauri, R. Zizza, On the power of circular splicing, Discrete Applied Mathematics 150 (2005) 51-66.

[5] P. Bonizzoni, C. De Felice, G. Fici, R. Zizza, On regularity of circular splicing languages: a survey and new developments, Natural Computing (2009), to appear.

[6] P. Bonizzoni, C. De Felice, R. Zizza, Circular languages generated by complete splicing systems and pure unitary languages (extended version), http://www.dia.unisa.it/professori/defelice/ssssss.pdf

[7] R. Ceterchi, C. Martin-Vide, K.G. Subramanian, On some classes of splicing languages, in: (N. Jonoska, G. Păun, G. Rozenberg Eds.) Aspects of Molecular Computing: Essays in Honor of the 70th Birthday of Tom Head, Lecture Notes in Computer Science 2950 (2004) 83-104.

[8] R. Ceterchi, K. G. Subramanian, Simple circular splicing systems, Romanian Journal of Information Science and Technology 6 (2003) 121-134.

[9] C. De Felice, G. Fici, R. Zizza, Marked systems and circular splicing, in: (E. Csuhaj-Varjú, Z. Esik, Eds.) Proc. FCT 2007, Lecture Notes in Computer Science 4639 (2007) 238-249.

[10] C. De Felice, G. Fici, R. Zizza, A characterization of regular circular languages generated by marked splicing systems, Theoretical Computer Science (2009), doi: 10.1016/j.tcs.2009.07.005. 
[11] A. Ehrenfeucht, D. Haussler, G. Rozenberg, Conditions enforcing regularity of context-free languages, in: Proc. ICALP'82, Lecture Notes in Computer Science 140 (1982) 187-191.

[12] A. Ehrenfeucht, D. Haussler, G. Rozenberg, On regularity of context-free languages, Theoretical Computer Science 27 (1983) 311-332.

[13] I. Fagnot, Simple circular splicing systems, Preproc. of Dixième Journées Montoises d'Informatique Théorique, 2004, Liege.

[14] E. Goode, D. Pixton, Semi-simple splicing systems in: (C. Martine-Vide, V. Mitrana, Eds.) Where Mathematics, Computer Science, Linguistics and Biology Meet, 343-357, Kluwer Academic Publ., 2001, Dordrecht.

[15] D. Haussler, Insertion languages, Information Sciences 31 (1983) 77-90.

[16] T. Head, Formal language theory and DNA: an analysis of the generative capacity of specific recombinant behaviours, Bulletin of Mathematical Biology 49 (1987) 737-759.

[17] T. Head, Splicing schemes and DNA, in "Lindenmayer Systems: Impacts on Theoretical Computer Science and Developmental Biology", Springer-Verlag, Berlin (1992) 371-383.

[18] T. Head, G. Păun, D. Pixton, Language theory and molecular genetics: generative mechanisms suggested by DNA recombination, in: (G. Rozenberg, A. Salomaa, Eds.) Handbook of Formal Languages, Vol. 2, 295-360, Springer Verlag, 1996.

[19] J.E. Hopcroft, R. Motwani, J.D. Ullman, Introduction to Automata Theory, Languages, and Computation, Addison-Wesley, Reading, Mass., 2001.

[20] M. Lothaire, Combinatorics on Words, Encyclopedia of Mathematics and its Applications, Addison Wesley Publishing Company, 1983.

[21] A. Mateescu, G. Păun, G. Rozenberg, A. Salomaa, Simple splicing systems, Discrete Applied Mathematics 84 (1998) 145-163.

[22] G. Păun, G. Rozenberg, A. Salomaa, DNA Computing, New Computing Paradigms, Springer-Verlag, Berlin, 1998.

[23] D. Pixton, Regularity of splicing languages, Discrete Applied Mathematics 69 (1996) 101-124.

[24] R. Siromoney, K.G. Subramanian, A. Dare, Circular DNA and splicing systems, in: Proc. of ICPIA, Lecture Notes in Computer Science 654 (1992) 260-273. 